\title{
EL LABERINTO \\ DEL DESARROLLO SUSTENTABLE
}

\author{
Carlos Mallorquin*
}

RESUMENः El texto intenta dar una descripción de la evolución teórica del trabajo de Enrique Leff sobre la cuestión ambiental durante varias décadas; subraya los itinerarios por medio de los cuales su vocabulario, habiendo iniciado desde el campo de la ecología y el marxismo, da pie a la producción de un discurso autóctono que desplaza a la mayoría de las nociones de procedencia europea y anglosajona culminando en un concepto del medio ambiente sui generis. A lo largo de la evolución teórica percibo una dislocación, del vocabulario en cuestión, de la problemática de las asimetrías de poder y las relaciones sociales entre las unidades productivas. Por lo tanto, en la segunda parte propongo una respuesta a dicha «falta» ofreciendo un matrimonio teórico con un discurso latinoamericano igualmente autóctono sobre la problemática de la economía y las asimetrías de poder, conocido como el estructuralismo latinoamericano.

Pala bras Clave desarrollo sustentable, ecología, medio ambiente, teoría latinoamericana, Enrique Leff.

\footnotetext{
* Docente-investigador de la Unidad Académica de Estudios del Desarrollo de la Universidad Autónoma de Zacatecas, México.
} 
ABSTRACT: This chapter attempts to describe the theoretical evolution of the work of Enrique Leff on the environmental question across several decades; it traces the evolution of his vocabulary, arising from the ecology camp and Marxism, gaining its footing in the production of an autochthonous discourse that supplants many earlier European and Anglo-Saxon notions, culminating in a sui generis concept of the «environment.» Throughout this theoretical evolution, a dislocation is detected in the vocabulary in question, of the problematic of power asymmetries and social relations among productive units. This leads, in the second part, to a proposed response to this «rupture», offering a theoretical marriage with Latin American discourse, equally autochthonous, on the problematic of the economy and power asymmetries, known as Latin American Structuralism.

KEY WORDS: sustainable development, ecology, environment, Latin American theory, Enrique Leff. 


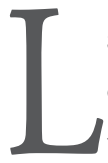

a evolución que ha sufrido el discurso sobre el desarrollo sustentable o del medio ambiente en las últimas décadas refleja los cambios y estrategias de las políticas para pensar el desarrollo en la periferia y en los centros industriales.

Ríos de tinta han recorrido el mundo después de la Conferencia en Estocolmo (1972). Surge la declaración en Cocoyoc en octubre de 1974 y el informe Dag Hammarskjold para el séptimo periodo extraordinario de sesiones de la Asamblea General de la Naciones Unidas, en septiembre de 1975, y la Comisión Mundial sobre Medio Ambiente y Desarrollo en 1987, así como El Informe Brundtland en 1987 o la Cumbre de la Tierra en Río de Janeiro en 1992. A su vez, en América Latina se dio una respuesta, primeramente en el idioma inglés, a la tesis sobre los límites del crecimiento en 1976: ¿Catástrofe o nueva sociedad. El modelo mundial? ${ }^{1}$

Esta sección de la introducción tiene el objetivo de ofrecer una breve reseña de los «andamiajes» (Leff, 2010a:272) básicos y caminos por los que «discurrió» Enrique Leff a lo largo de más de «dos nadas» (Leff, 2010a: 256), ya que como dice el tango, «veinte años» no son «nada». Le sigue un apartado, «Disciplinas, saberes y rupturas teóricas», donde ofrecemos algunos de los planteamientos y vocabulario de Leff en torno al desarrollo sustentable y la problemática ambiental, finalizando con la sección que proyecta, de nuestra parte, un apoyo teórico para pensar la nueva racionalidad ambiental y productiva que formula Leff ( $«$ Los excedentes del poder»).

Los cambios y giros conceptuales dan cuenta de la lucha por construir cierta hegemonía y práctica en materia de distribución y utilización de los recursos productivos. Los planteamientos de la obra de Leff parten inicialmente del campo disciplinario de la ecología y sus diversas corrientes, provenientes del discurso hegemónico europeo y anglosajón. Discursos que serán «superados» (en el sentido hegeliano) culminando

${ }^{1}$ Se agradece a Guillermo Foladori el hacerme llegar el texto. 
en una teorización propia y latinoamericana. No obstante, sus importantes logros y transformaciones teóricas, en los últimos años (Leff, [2006] 2011), la explicación y evolución de sus ideas convocan una vez más a algunos exponentes del pensamiento continental y a cierto vocabulario de Heidegger. Creo que esta envoltura debe recibir cierta vigilancia teórica para impedir que los frutos teóricos de Leff no se conviertan en obstáculos prácticos y políticos para estrategias alternativas de la organización productiva y las de sus presupuestos, así como sus diversas lógicas y mecanismos constitutivos.

La transición teórica de pensar (una) lógica del capital en general, y sus efectos destructivos sobre los recursos productivos y su distribución asimétrica, tanto en costos ambientales como en el de sus recompensas, para luego tomar un giro y esfuerzo teórico que propone maneras de recuperar perspectivas que redunden en análisis específicos, supondrá marginar esa visión y lógica unitaria, lo cual conduce a observar configuraciones sociales regionales, sobre las alternativas prometidas de las transformaciones productivas, y que son los elementos teórico-políticos que deben rescatarse.

No obstante, las transformaciones teóricas, paradigmas y conceptos, encontramos que algunas vías de resolución conceptual pueden generar cierta paralización de la política y de la acción en torno a la reconstitución del tejido del medio ambiente dados los efectos negativos hacia el mismo generadas por las actuales políticas. La obra de Leff presenta un caso singular, que por sus esfuerzos teóricos, que ya casi superan el medio siglo de reflexión en el campo del desarrollo sustentable o del medio ambiente, vale la pena subrayar: una teorización y mirada regional, latinoamericana.

Como toda obra, producto de transformaciones teóricas y políticas durante varias décadas, presenta aspectos fructíferos para pensar la problemática de la organización de la producción y del desarrollo sustentable. Asimismo, intentaremos ofrecer una opción para fundamentar la propuesta y no caer en el dilema de la sustentabilidad como un oxímoron sin alternativas específicas.

La tarea se facilita si se elude el panorama de la pluralidad de los discursos sobre el desarrollo sustentable, a partir de distintos puntos de 
partida, y ubicar la perspectiva desde la(s) perspectiva(s) de un autor, o teórico, lo que a su vez allana la observación de los desfases teóricos y obstáculos que limitan algunos de los postulados de su perspectiva sobre la problemática del desarrollo sustentable y del medio ambiente.

Desde la ecología, vemos su crítica y transición conceptual a dicha disciplina, muy singular: el ecomarxismo y el materialismo histórico, para resolver la articulación entre la cultura/naturaleza; su conocimiento sobre el «manejo integrado de los recursos naturales»; la cadena trófica, productividad primaria y secundaria; la recuperación de la etnobotánica y etnociencias y las culturas prehispánicas para pensar alternativas de regeneración del sustrato productivo y uso de sus recursos.

Asimismo, es muy esclarecedora la visión que su obra presenta sobre la historia y teorización de las limitaciones de las disciplinas y sus compartimentos estancos; la función de la inter e intradisciplinariedad como obstáculos y apoyo a un proceso de teorización, ${ }^{2}$ que suponen nuevas perspectivas u objetos del conocimiento, lo cual redundó finalmente en una concepción sobre el medio ambiente muy singular.

La recuperación de los movimientos sociales, indígenas, étnicos y campesinos para el movimiento del desarrollo sustentable, y sus visiones de reapropiación de la naturaleza y reconfiguración del proceso productivo, intentan disipar las dos lógicas opuestas, la del valor mercantil y la de la ética comunitaria, lo cual obliga al propio Leff a desarrollar una perspectiva teórica alternativa, aportando ciertos elementos teóricos y prácticos para la misma.

${ }^{2}$ Véase, por ejemplo, los dos primeros capítulos de Leff (2007). Muchos años después de haber iniciado el proyecto, dice: «El proyecto interdisciplinario que se funda en la ecología - como ciencia por excelencia de las interrelaciones-, que se inspira en el pensamiento de la complejidad basado en una ecología generalizada para articular las diferentes disciplinas y campos de conocimiento, mantiene una voluntad unificadora, sin indagar los obstáculos paradigmáticos y los intereses disciplinarios que resisten e impiden tal vía de retotalización holística del saber. Este proyecto interdisciplinario fracasa en su propósito de crear una ciencia ambiental integradora, de ofrecer un método para aprehender las interrelaciones, interacciones e interferencias entre sistemas heterogéneos, y de producir una ciencia transdisciplinaria superadora de las disciplinas aisladas» (Leff, 2010a: 177). 
Inquieta, sin embargo, que los resultados de la transición teórica presenten una creciente marginación de la propuesta sobre la lógica productiva o racionalidad productiva. ${ }^{3}$ Mientras elaboraba la crítica al ecomarxismo y las perspectivas holísticas y sistémicas sobre la racionalidad ambiental y la postulación de la construcción imperativa de una nueva economía o racionalidad ambiental alterna, Leff criticaba los efectos negativos del capital, a partir del marxismo y simultáneamente iniciaba una búsqueda de otra perspectiva para comprender las lógicas, en plural, del capital y, por lo tanto, la organización de la producción, las formas de propiedad o lo que yo llamaría las «formas de posesión en separación» de las condiciones de existencia de las unidades productivas, obligándolo a generar una explicación diferente. Una vez que se va desplazando la noción de la teoría del valor marxista, ${ }^{4}$ y del capitalismo en general, reivindicando la posibilidad de organizar o gestionar los recursos productivos («naturales»), y las unidades productivas por medio de la teoría del ecodesarrollo y el ecotecnodesarrollo; la dicotomía sociedad/naturaleza, la ecologización de la teoría, se facilita observar la importancia de tomar en cuenta las culturas y prácticas específicas de las comunidades rurales e indígenas, sin advertir que marginaba elucidaciones sobre las especificidades de las relaciones que dan cuenta de las asimetrías de poder y las prácticas destructivas del capital.

De hecho critica algunos modelos del ecodesarrollo debido a que no están necesariamente en contraposición a la lógica del capitalismo. Es en esta etapa cuando vemos aparecer la nueva concepción sobre el medio ambiente y la importancia de pensar las luchas de las comunidades como la «reapropiación de la naturaleza».

${ }^{3}$ Leff insiste que desde « 1975 hemos venido proponiendo la idea de que otra economía es imperativa y es posible» (Leff, 2010a: 83).

${ }^{4}$ En 1982 presentaba una de sus primeras críticas a la teoría del valor-trabajo, pero todavía se encuentra con un pie dentro y otro fuera, es decir, no ha logrado desplazar la noción ya que su interrogante se remite a las consecuencias teóricas de la noción cuando predomina la robótica tecnológica y del chip en los procesos de trabajo. Pero se sabe que aunque la proporción de la fuerza de trabajo «viva» sea mínima en la composición orgánica del capital, de lo que se trata — diría Marx - es del grado de explotación o tasa de extracción del plusvalor (Leff, 1982). La ruta y el desplazamiento plena de la teoría objetiva o valor-trabajo puede verse en el primer capítulo de Leff (2013 [2004]). 
Es entonces que surge cierta ausencia, cierta falta de explicación que considero «incolmable» que tratan los aspectos del poder y su transformación: las formas de posesión en separación de las condiciones de existencia de las unidades productivas, espacios e intersticios donde se generan las asimetrías de poder que imponen ciertas lógicas productivas. Sin duda Leff no deja de señalar la importancia de los nuevos movimientos y luchas de las comunidades por resistir al capitalismo y buscar alternativas a través del movimiento ambiental, o sus similares ideológicos. Es allí cuando la aparición de la teoría del medio ambiente y del saber ambiental tiende a olvidar los aspectos que exigen una explicación más específica sobre las condiciones asimétricas de poder entre unidades productivas y regiones. Este ámbito de las relaciones sociales, como propondremos al final, no requiere necesariamente una teoría general del valor (marxista o neoclásica), sino una teoría del poder desarrollada por el pensamiento estructuralista latinoamericano, donde las asimetrías del poder y por tanto la heterogeneidad, cultural y económica, son consecuencia de la manera en que se poseen en separación, algunas condiciones de existencia de las unidades productivas.

\section{DISCIPLINAS, SABERES Y RUPTURAS TEÓRICAS}

Un poquito de ecología es necesario para comprender la vía que toma la evolución de las reflexiones de Leff, ya que supondrá su desplazamiento, y subsunción, al igual que los discursos sobre el desarrollo sustentable, bajo una problemática más amplia del vocabulario en torno al ambiente y su crisis, y los discursos de la complejidad ambiental.

A partir del canon básico de algunos principios de ecología, podemos leer que a mediados del siglo xix, Haeckel define la ecología como el «conocimiento que trata de las relaciones entre los organismos y su medio ambiente inorgánico y orgánico, como la economía de la naturaleza» (Gallopin, 2000-1986: 88):

[... el concepto clave que subyace a todas ellas es el de interrelación; la ecología se concentra primariamente no en los organismos en sí mismos ni en el 
ambiente de los organismos por su propio interés, sino en las interrelaciones entre los organismos y sus ambientes, y su objetivo central es describir los principios que gobiernan esas interrelaciones [...] [;] es en la caracterización del sistema ecológico objeto de estudios donde yacen las diferencias. Organismos, poblaciones, comunidades, ecosistemas, naturaleza, son propuestos como candidatos para circunscribir la unidad de estudio ecológico [...] Un sistema ecológico no tiene una dimensión o escala única; podemos distinguir sistemas ecológicos de todos los niveles desde el organismo hasta la ecosfera. Lo que caracteriza a un sistema como ecológico no es su dimensión o grado de agregación, sino su naturaleza [....

Un sistema en general puede ser definido como un conjunto coherente de elementos interactuantes o interdependientes. Todos los sistemas biológicos son sistemas abiertos, caracterizados por un continuo intercambio (Gallopin, 2000-1986: 89-90 subrayados del autor). ${ }^{5}$

Es instructivo recordar la noción de ambiente y sistema engendrada por dicha definición y en relación a la evolución de las ideas de Leff, porque serán precisamente esas categorías las que serían transfiguradas en conceptos muy específicos.

Si bien el vocabulario de Leff tiene raíces en la ecología en torno a la práctica sobre la gestión o manejo integrado de los recursos naturales, lo hace desde una crítica de los mismos, inicialmente intentando buscar formas de subsumir y desplazar el discurso a partir del objeto del materialismo histórico: el modo de producción capitalista. ${ }^{6}$

${ }^{5}$ «Un sistema ecológico consiste de uno o más organismos, junto con los varios componentes del ambiente con los que están relacionados funcionalmente. Los componentes de los sistemas ecológicos son por lo tanto elementos (organismos, grupos de organismos -incluyendo los seres humanos- componentes abióticos, etcétera) y las relaciones (interacciones de alimentación, competencia, regulación, reacciones a variables ambientales, etcétera)» (Gallopin, 2000-1986: 90, subrayados del autor).

${ }^{6}$ No hay lugar aquí para discutir la lectura y adecuación de Leff sobre dicha problemática, pero la estrategia teórica está «marcada» por la discusión de Althusser (2004 [1967]) y discípulos, y es la que impera inicialmente por esa época en las ideas de Leff, no sólo en lo que se refiere a la concepción del modo de producción, sino en la manera en que reflexiona sobre el saber, la(s) teoría(s) y su(s) transformación(es); la distinción entre el objeto del conocimiento y lo real, que a su vez sustenta la evaluación, pertinencia y alcance de los proyectos intra e interdisciplinarios para la 
No cree posible que la ecología pueda conceptualizar la problemática del ambiente y gestión de los recursos productivos, es decir, su administración u organización, ya que no toma en cuenta las relaciones sociales en que se encuentran inmersos. Sin embargo, menciona regiones/ territorios que presentan cierto potencial ambiental y que dependen de su estructura ecosistémica, y procesos productivos, por la existencia de ciertas tradiciones y las prácticas de las comunidades a través de sus valores culturales. Es así que hace surgir la dicotomía sociedad-naturaleza, relación dinámica y articulación de un proceso tecnológico y cultural que la gestión ambiental del desarrollo tiene por obligación pensar: las relaciones y organización del manejo integrado de los recursos, o sea, la racionalidad ambiental.

La complejidad ambiental del socioecosistema requiere de varios nuevos objetos teóricos, todo lo cual lleva a la reconstrucción de la ecología o sus conocimientos actuales. Objetos de una o más ciencias que no pueden asimilarse. Las condiciones históricas y económicas del objeto de la ecología supone su internalización a través de las ciencias sociales y no por medio de una «ecologización» de los «procesos sociales» (Leff, 2007: 95).

A su vez, el territorio regional condiciona las prácticas sociales, y por tanto la «conformación del medio incide en la constitución y evolución de las culturas y en sus desarrollos técnicos» (Leff, 2007: 95), quienes se apoyan en su «estilo étnico de aprovechamiento del ambiente por una cultura» que «está sobredeterminado por su estado de dependencia y dominación» (Leff, 2007: 95).

Por lo tanto, la «gestión ambiental del desarrollo, fundada en el potencial ecológico y en la conservación de la diversidad de estilos culturales» (Leff, 2007: 95-96) supone explicar una formación social, la cual está articulada bajo una diversidad de procesos ecológicos, culturales e históricos.

generación de un saber sobre el medio ambiente; en particular, véase capítulo 2 (Leff, 2013 [2004]) y los tres primeros capítulos en Leff (2007 [1986]). 
El propio ecomarxismo no ofrece soluciones teórico-prácticas para generar una racionalidad social alternativa, de donde cabe pensar el concepto de racionalidad ambiental. El marxismo es demasiado «esquemático» para comprender los procesos «ecológicos, tecnológicos y culturales» (Leff, 2007: 277). Según Leff, para el marxismo el ambiente es más que un elemento, un «costo». La teoría ecomarxista quiso ir más allá de la unificación de la ecología y del marxismo, incluso intentó un proyecto de incorporar el ámbito natural. El debate sobre la destrucción de sus propias condiciones de existencia por la lógica depredadora del capital, o sea, de los recursos naturales, hizo pensar la segunda contradicción del capital: "autodestrucción y el creciente costo de las condiciones de producción del capital» (Leff, 2007:341). ${ }^{7}$

Sin embargo, el ambientalismo es una fuerza social, con la correspondiente capacidad para frenar los procesos «ecodestructivos», siendo el ecomarxismo una estrategia que solamente se concentró en aquellos aspectos que conceptualizan «los condicionantes sociales de la praxis» (Leff, 2007: 363), en lugar de una estrategia para el cambio social:

[...] orientar la reflexión del movimiento hacia su propia práctica, de lo cual podrán surgir las estrategias para generar un poder real de transformación social [...] [y] podrá desbloquear la ortodoxia paralizante y desencadenar estrategias de organización de acción, capaces de concretar el potencial innovador del ambientalismo mediante las oportunidades que ofrece el campo cambiante del poder (Leff, 2007:363).

Es que para entonces Leff observa que el ámbito de lucha teórica y política ha transitado hacia el «campo conflictivo de las relaciones sociedad-

\footnotetext{
${ }^{7}$ Cabe mencionar que Leff no es ningún aprendiz de marxismo, conoce y ofrece todos los sinsabores de una lectura profunda de Marx, tanto de sus textos publicados en vida como los manuscritos subsecuentes, así como los debates sobre la teoría del valor en la agricultura, recuérdese la famosa cita de Marx cuando declara inoperante la noción de valor en una economía debido al avance de la tecnología: «el tiempo de trabajo cesará de ser la medida del trabajo, así como el valor de cambio dejará de ser la medida del valor de uso» (citado en Leff, 2007: 342; véase también, G. Foladori, 1996; Mir y Gorostiza, 2000).
} 
naturaleza» (Leff, 2007: 363), lo cual se presenta como una contraposición entre capitalismo ecológico y un proyecto socialista ecológico y democrático. Ha surgido la defensa y construcción de una racionalidad ambiental en términos del concepto de productividad ecotecnológica lo cual suponía el análisis de las «condiciones ecológicas, tecnológicas, económicas y culturales»:

El ambientalismo plantea la posibilidad de construir una nueva racionalidad productiva. Ésta se basa en la concepción del ambiente como un sistema potencial y un potencial productivo, a partir de la activación de los principios de una productividad ecotecnológica generada por la articulación de la productividad ecológica de los recursos naturales, la productividad tecnológica de sus procesos de transformación y la productividad social de la organización productividad de las comunidades (Leff, 2007:397, subrayados del autor).

Ello haría posible la generación de un paradigma alternativo de producción, fundamentado en el concepto de productividad ecotecnológica, el «cual articula los niveles de productividad ecológica, tecnológica y cultural en el manejo integrado de los recursos productivos» (Leff, 2007: 104). Obviamente esta lógica productiva no se evalúa en términos de los precios del mercado, y valoriza positivamente ciertos recursos en lugar de otros. De esta manera se intentan preservar ciertos recursos potenciales que han sido ignorados por su bajo valor de cambio (Leff, 2007: 105) en términos monetarios.

Según Leff, las «complejas interrelaciones» entre esos niveles de productividad social suponen una conceptualización «sincrónica y diacrónica de los procesos culturales, ecológicos y tecnológicos que la constituyen, que sirva como soporte del manejo integrado de los recursos naturales y sociales para un desarrollo económico sostenido» (Leff, 2007: 106). Existe un efecto sistémico de generación de nuevos potenciales productivos (Leff, 2007: 107) a raíz de una nueva articulación de los recursos naturales, proceso tecnológicos y valores culturales. El conocimiento de los «diferentes niveles de articulación de sus procesos materiales», conduce a una estrategia 
conceptual para construir una «racionalidad ambiental» cuyas bases se deben a una «racionalidad social alternativa» (Leff, 2007: 107) a la capitalista. Por tanto, el «concepto de productividad cultural permite dar cuenta de la fuerza productiva de una comunidad, a partir de su percepción y de las formas de aprovechamiento productivo de sus recursos, de sus motivaciones y de asimilar nuevos conocimientos a sus prácticas productivas tradicionales» (Leff, 2007: 112).

En dicho caso, la productividad no se puede medir o evaluar en términos de índices o agregados monetarios. La dimensión cultural sobre un proceso sostenido de desarrollo solamente puede medirse en términos de los valores dominantes de las comunidades en cuestión.

Dado el caso, Leff retorna una vez más a la problemática de los préstamos e interrelaciones de conceptos en estudios interdisciplinarios para hablar de la dispersión de sentidos de los conceptos. Lo cual hace posible reflexionar sobre «diferentes perspectivas de análisis de la problemática ambiental», a partir de las «relaciones de poder (del poder real y del saber del poder)» (Leff, 2007: 119).

La degradación del medio ambiente, dice Leff, y la destrucción de los recursos naturales no ha generado su «adecuada conceptualización» mediante la inscripción de los procesos naturales en términos «sociohistóricos» (Leff, 2007:124). Si bien la propuesta inicia criticando las debilidades de la disciplina de la economía en su intento por «integrar las externalidades de los procesos productivos, tampoco la sociobiología ha logrado integrar la dicotomía sociedad-naturaleza», aspecto irresuelto también desde la ecología cuando incorpora a la «sociedad» como subsistema del «ecosistema global» (Leff, 2007: 124).

Hasta entonces, las reflexiones de Leff se podían ubicar dentro del contexto de las corrientes del ecodesarrollo, lo cual pronto también presentará limitaciones para pensar la nueva racionalidad ambiental o racionalidad productiva:

La estrategia del ecodesarrollo se funda pues en una reorganización productiva que integra los niveles de productividad natural y tecnológica. La primera debe fundarse en la conservación de las estructuras ecológicas básicas que 
garantice la productividad sostenible de los recursos naturales; en la intervención de una tecnología ecológica que modifique el ecosistema [... L La productividad ecotecnológica debe asegurar un aumento en la eficiencia termodinámica de los procesos de transformación industrial, adecuando los diferentes recursos energéticos al tipo de necesidades, incrementando la productividad ecológica de los ecosistemas y desarrollando nuevas fuentes energéticas basadas en recursos inagotables como la energía solar (Leff, 2007: 222).

El propio Leff intenta explicar teóricamente este giro conceptual:

El concepto de racionalidad ambiental se circunscribe así, en este momento, al campo de la producción. Emerge de una crítica de la economía política del ambiente y se orienta hacia una transformación de la racionalidad productiva. Rompe con la concepción reduccionista del hombre en su función como fuerza de trabajo en la racionalidad económica dominante, para inscribir los potenciales de la naturaleza y de la cultura en los procesos productivos. La racionalidad ambiental combate así al proceso ideológico que ha universalizado la dimensión del trabajo, de las necesidades y de la producción, abriendo posibilidades a la construcción de nuevos «modos de producción». Sin embargo, el concepto de racionalidad ambiental tiene un sentido más amplio, que concierne a los valores de la democracia, al campo del poder y a los sentidos de la existencia del hombre. Con el concepto de calidad de vida, el ambientalismo apunta hacia una racionalidad económica y de la racionalidad teórica que, como su espejo, se han constituido en la cultura de la modernidad, orientando la construcción de una nueva racionalidad social (Leff, 2007: 242, subrayados míos).

Es sintomático el cambio teórico y de perspectiva; va incorporando cuestiones que se alejan o marginan relativamente aquellos aspectos de la organización del proceso de trabajo y la tecnología para culminar en aspectos que tienen que ver con las asimetrías del poder:

Dentro de diferentes estructuras de poder, con distintos patrones de distribución de la riqueza y medios para ofrecer un bienestar social a la población, la producción, la producción de satisfactores se ha fundado en una racionalidad 
económica de corto plazo [... $]$ sin embargo, la productividad primaria de los ecosistemas - su capacidad natural para elaborar materia vegetal mediante el proceso fotosintético y su potencial para producir valores de uso naturalesestá excluida de los principios de productividad económica, así como los costos ecológicos del crecimiento no son considerados en la contabilidad económica. Los procesos naturales que soportan el stock de recursos no se integran en la evaluación del producto nacional [...] proceso productivo [que] se caracteriza por el desajuste entre las formas y ritmos de extracción, explotación y transformación de los recursos naturales y las condiciones ecológicas para su conservación y regeneración (Leff, 2007:243).

El ecodesarrollo surgió durante la lucha por definir un nuevo orden mundial, en el contexto del Informe del Club de Roma en 1972 y los «límites del crecimiento», y las insuficiencias de la perspectiva teórica de la economía. Según Leff, se puede observar en la Declaración de Cocoyoc de octubre de 1974 y el informe Dag Hammarskjold para el séptimo periodo extraordinario de sesiones de la Asamblea General de la Naciones Unidas, en septiembre de 1975.

Sus limitaciones tienen que ver con las «determinaciones que imprime un modo de producción específico o una cultura en su medio ambiente» (Leff, 2007:318), y tiende a «refuncionalizar la racionalidad económica, incorporando una "dimensión ambiental" a las políticas del desarrollo» (Leff, 2007:319).

Sin embargo, la propuesta del ecodesarrollo de promover la autonomía cultural y «gestión tecnológica de las comunidades» (Leff, 2007: 319) no cuestionó la factibilidad de que sus objetivos se pudieran realizar «dentro de los paradigmas económicos y las estructuras institucionales prevalecientes» (Leff, ídem.). No cambió tampoco cuando sus propósitos se definen como la «valorización de los recursos específicos de cada ecoregión» (Leff, 2007:320) que solamente promovería la diversificación de «los procesos técnicos de explotación de la naturaleza»(Leff, 2007:321). ${ }^{8}$

${ }^{8}$ El autor más reconocido sería Sachs, de hecho fue profesor de Leff a mediados de los sesenta. 
El entonces consenso mundial buscaba una solución a la crisis ambiental, lo cual generó básicamente dos corrientes, una se deriva de la Conferencia Mundial sobre el Medio Ambiente y Desarrollo, en Río de Janeiro 1992 y la Agenda 21. Según Leff, cohabitaron la perspectiva del discurso del desarrollo sustentable que proponía «la reconversión ecológica de la racionalidad económica vía la liberación del comercio» (Leff, 2007: 327) por medio del mercado; la otra insistía en la gestión participativa de los recursos y democracia ambiental, y la reconstrucción de una nueva racionalidad social con la participación de directa de la población. El ecodesarrollo y el desarrollo sustentable luchan por definir el concepto mismo de desarrollo alternativo: una intenta la «regeneración de los recursos naturales y de equilibrio ecológico» (Leff, 2007:331) y la otra insertarse «en las luchas sociales por la autogestión productiva de las comunidades y la construcción de una racionalidad ambiental» (Leff, 2007: 331-32), son las disyuntivas donde una de ellas se inserta en las luchas de las condiciones de producción de las comunidades indígenas.

Vemos que Leff incorpora la importancia de los movimientos sociales en la reconstrucción del discurso en torno al desarrollo de los recursos sustentables:

Sin embargo, al mismo tiempo que las estrategias del ecodesarrollo y los objetivos del desarrollo sustentable pueden asimilarse a las estrategias de respuesta del capital a las condiciones del cambio global (incorpora propuestas que, para realizarse, requieren un conjunto de transformaciones institucionales, jurídicas y políticas (Leff, 2007: 320).

Lo cual, según Leff, generaron movimientos y luchas anticapitalistas, pero en los hechos:

[... ] la falta de un análisis político del campo contradictorio en el que se insertan las estrategias del ecodesarrollo - y el discurso más actual del desarrollo sustentable - ha favorecido el surgimiento de confusiones y suspicacias en torno a sus propuestas (Leff, 2007:321). 
Es este aspecto estratégico político el que va tomando la delantera en la obra de Leff: «los movimientos de reivindicación», política y cultural de los indígenas y zonas rurales. Esta perspectiva más allá de la lógica y criterios de una «economía de la contaminación» y de protección de los ecosistemas $\mathrm{y}$ «medio ambiente». Y por tanto la

[...] problemática ambiental es una cuestión eminentemente política. Ello se refleja en la emergencia de movimientos sociales de defensa de los recursos, las tierras y los valores culturales de los pueblos [... E El movimiento ambientalista induce una fuerza social que, como presión política, incrementó el precio de los recursos y de los servicios ambientales, aumentando los costos privados del capital [... ] ese mecanismo de «ajuste de cuentas» mediante la expresión de intereses está limitado a la correlación de fuerzas puestas en juego [...] Pero, aun en el caso de que el movimiento ambiental consolidara sus fuerzas, su sentido no se agotaría en ser un eficaz transmisor de los valores inconmensurables del ambiente hacia la estructura de precios del mercado y costos del capital. Lo más sustantivo del movimiento ambiental es justamente su orientación hacia la construcción de una nuevo paradigma productivo y de una nueva racionalidad social (Leff, 2007: 270-71). ${ }^{9}$

Una vez inspeccionado la problemática del marxismo y el ecomarxismo, Leff cree que existen bases para "plantear un paradigma productivo alternativo al de la racionalidad económica dominante» que dependería más de satisfactores básicos, en la «socialización del acceso» y «apropiación de la naturaleza», «descentralización» y ordenamiento «ecológico de las actividades productivas»y las «comunidades en la gestión de los recursos ambientales» (Leff, 2007:358). Se trata, dice Leff, de un "paradigma» en el que la «naturaleza» y la «cultura» «no entran tan sólo como procesos mediadores, como condición o soporte de la producción, sino como trabajo social y fuerzas productivas directas» (ídem.), en el sentido del modelo «ecotecnólógico» «para el manejo integrado, sustentable y sostenido de los recursos naturales, fundado en el concepto de racionalidad ambiental»

9 Ver también, capítulos 7-8-9 y 10 en (Leff, 2010). 
(Leff, 2007: 358). Aquí, la propia noción de cultura y naturaleza son parte de las fuerzas productivas.

Es en este instante cuando Leff plantea un cambio teórico crucial sobre la complejidad ambiental y su objeto, cuyos efectos fueron paralizadores, en términos políticos, debido a que la problemática de organizar la unidad productiva o el territorio y sus regiones, a partir de la lucha por parte de las comunidades por «apropiarse» de sus recursos productivos o condiciones de existencia - Leff, dice la «naturaleza»-, va siendo marginada con la teorización del «ambiente».

Como transcribiendo su propia evolución teórica, Leff dice que el materialismo histórico transita desde una perspectiva crítica al modelo de acumulación de la degradación ambiental a la reelaboración de las categorías de naturaleza y cultura, situándolas en el centro mismo del «proceso productivo [...] [para] construir una nueva teoría de la producción (una nueva racionalidad productiva), que, más allá de considerar el ambiente como externalidad [...] incorpore el ambiente como potencial al proceso productivo» (Leff, 2007:335, subrayados el autor). Nótese que en esta ocasión el ambiente forma parte del proceso productivo. Asimismo, las categorías

$[\ldots]$ generales de naturaleza y trabajo no permiten aprehender la especificidad de las relaciones entre una formación social específica y su ambiente [...] de traducir la organización y los valores culturales en principios de productividad y de organización productiva en el manejo sustentable de los recursos naturales (Leff, 2007: 339). ${ }^{10}$

De esta manera Leff transita hacia el último paso para dar por inapropiados los modelos sistémicos y holistas para pensar la organización

${ }^{10}$ «Desde que la naturaleza se convierte en un conjunto de objetos - dice Leff-y de procesos de trabajo, desde que los procesos ecológicos participan en la formación de valor y en la producción de plusvalor, lo natural se absorbe en el objeto del materialismo histórico; es decir el proceso de reproducción del capital. El recurso natural no es producto de un metabolismo biológico; la fuerza de trabajo no es el desgaste energético de los hombres [...] implican el soporte de las leyes biológicas de los organismos vivos; pero no son entes naturales cuya existencia sea independiente de los procesos históricos. [...] Por lo anterior, la articulación entre naturaleza y sociedad no puede explicarse como un simple intercambio entre la cultura y su medio ambiente» (Leff, 2007: 139). 
los recursos naturales y ambientales, que como veremos genera cierta marginación del planteamiento en torno a las formas en que la comunidad o unidades productivas poseen en separación ciertas condiciones de su existencia. El hecho que Leff sostenga posible una racionalidad productiva distinta, entre - a pesar de tratarse de lógicas contrapuestas - las unidades productivas tradicionales, bajo patrones de comportamiento específicos, y las que se guían por el mercado (ya no habla del «valor»), da fe de la posibilidad para enarbolar políticas alternativas: "plantear un paradigma productivo alternativo al de la racionalidad económica dominante» que dependería más de «satisfactores» básicos, en la «socialización del acceso» y «apropiación de la naturaleza», «descentralización» y ordenamiento «ecológico de las actividades productivas» y las «comunidades en la gestión de los recursos ambientales» (Leff, 2007: 358).

Pero previamente fue necesario haber despachado el aporte holístico de la relaciones sociedad-naturaleza del marxismo y considerar las relaciones «sociedad-naturaleza dentro de las relaciones sociales de producción» politizando el ambiente, que «aparece como un objeto complejo y polivalente que abre opciones para transformar la racionalidad económica dominante y construir una racionalidad productiva sobre bases de equidad social y sustentabilidad ecológica» (Leff, 2007:35657). Esto da pie a una apertura teórica hacia al pensamiento complejo. Pero supone superar los enfoques sistémicos que conciben al ambiente:

[... ] como sistema externo al sistema económico, para estudiar los procesos de coevolución entre sistema económico y sistemas geoambientales. No se trata de hacer desaparecer el ambiente subsumiéndolo en la capitalización de la naturaleza, ni mantenerlo como un sistema externo a la esfera económica, sino de integrar el ambiente en la producción, no sólo como condición, sino como potencial y fuerza productiva; de pensar en el ambiente como una articulación de procesos culturales, ecológicos tecnológicos y económicos (Leff, 2007: 357).

Es que pareciera ser que la noción del ambiente toma una ubicuidad como totalidad contradiciendo las posturas de pensar las estrategias 
productivas alternativas en plural. Este giro puede traducirse en cierta limitación conceptual, lo cual no sería un grave atolladero sino tuviera consecuencias para delimitar y proponer una racionalidad ambiental alternativa con su correspondiente racionalidad substantiva particular. Desde 1986, Leff ya denota una búsqueda de un vocabulario alternativo; menciona un interés por repensar la problemática ambiental:

[... el ambiente para los países latinoamericanos, más que límite para su desarrollo o lugar de disposición de desechos, aparece como un potencial ecológico $y$ cultural de producción de recursos naturales a partir de las condiciones geográficas que permiten generar altas tasas de productividad ecológica y a través de la diversidad de estilos étnicos de nuestros pueblos para su aprovechamiento. El ambiente no implica un costo que debe deducirse de las inversiones para el crecimiento económico, sino un potencial productivo para sostener nuevas alternativas de desarrollo [... cómo diferentes disciplinas (la ecología, la geografía, la antropología, la sociología, la economía, la arquitectura, el derecho, etcétera), dentro de su especificidad disciplinaria, interactúan con una concepción de lo ambiental, generando un proceso de internalización, intercambio y producción de conceptos que generan una reformulación de los contenidos de las disciplinas (2000: [1986] XIV, XIX).

De allí en adelante presenciamos el inicio de un «salto mortal» teórico conceptual — parafraseando a Marx - donde se aterriza con otra noción:

El ambiente no es simplemente un referente empírico, el punto de confluencia de disciplinas diversas o el trait d'union entre sociedad y naturaleza. El ambiente es el campo de externalidad de las ciencias que desde la potencia de su negación produce un saber que problematiza al conocimiento, generando condiciones teóricas para pensar la articulación de procesos de diversos niveles de materialidad y esferas de racionalidad (Leff, 2000: XXX, subrayados míos).

La noción del ambiente se va dilatando ${ }^{11}$ a tal punto que ha desplazado la idea de algún aspecto de colindancia entre entidades, de un adentro

${ }^{11}$ Según Leff, la «democracia ambiental» requiere un proceso de «transición» para «constituir una nueva economía, fundada en la integración de espacios de autogestión para el manejo integrado de 
y un afuera, que podrían pertenecer a dicho espacio o formar parte de su vecindad. Leff ofrece una breve historia:

[... el concepto de medio está implícito en el objeto de la biología evolutiva, de la antropología estructural y de la economía política. Este concepto surgió explícitamente del campo de la organización biológica que caracteriza el fenómeno vital al ser importado por Lamarck de la mecánica newtoniana. La noción de medio allí aparece como el éter o el fluido intermediario entre dos cuerpos, más tarde se transformó en el entorno o en el ambiente conformado como un sistema de conexiones que circundan y engloban a los centros organizadores de ciertos procesos materiales (biológicos, económicos, culturales). Es este sentido mecanicista del medio el que ha sido asimilado por los enfoques holistas del pensamiento ecologista actual (Leff, $2007: 87$, subrayados míos). ${ }^{12}$

Pero, por otra parte, surgen dudas sobre la noción, ya que el concepto de ambiente:

[... ] trata, más que [de] una dimensión, una variable o un espacio de integración de los saberes constituidos, es un proceso de transformación del conocimiento impulsado por una crisis de racionalidad económica e instrumental de la modernidad. Más que un paradigma omnicomprensivo del saber, una ecologización del conocimiento, un método general para el desarrollo de las ciencias [...] la cuestión ambiental, desde una perspectiva crítica, a toda una pléyade de conocimientos teóricos y técnicos [...] El saber ambiental está en proceso

los recursos en los niveles local y provincial: esto permitiría, tanto la articulación de mercados regionales e intercomunitarios, como la canalización de excedentes a los mercados nacional y mundial» (Leff, 2007: 400). De esta manera, Leff se olvida de la ley del valor para poder proponer la «articulación» entre diversos espacios y unidades productivas. A través de una «compleja concertación de intereses» (Leff, 2007: 400), donde se conjugan, Estado, mercado, y decisiones autónomas de autoridades y grupos locales, y la autogestión de las «comunidades urbanas y rurales» (Leff, 2007: 400). «La gestión ambiental no es el retorno romántico de la contemplación ecológica, o una utopía posmoderna desconectada del conflicto entre clases y de las bases materiales de la producción» (ídem).

${ }^{12}$ «El medio - dice Leff- puede reabsorberse en el sistema y el sistema puede convertirse en un ecosistema generalizado. Así se ha concebido el «ambiente humano» como el campo interdisciplinario de las «ciencias ambientales» en el que las externalidades ecológicas y sociales serían internalizadas en el terreno de las prácticas de la planificación» (Leff, 2007: 92). 
de gestación, [...] emerge de un proceso transdisciplinario de problematización y transformación de los paradigmas dominantes del conocimiento; trasciende las teorías ecologistas, a los enfoques energetistas y a los métodos holísticos en el estudio de los procesos sociales (Leff, 2010: 183). ${ }^{13}$

Pareciera ser que el saber ambiental ocupa un espacio y una perspectiva transcendental muy elusiva. ${ }^{14}$ Son «categorías que funcionan como estrategias conceptuales, que se construyen y se concretan a través de múltiples interrelaciones» (Leff, 2010: 195, subrayados del autor). También supone que si el ambiente puede pensarse, dice Leff, como una espacio de «articulación de procesos de diferentes órdenes de materialidad y racionalidad» (Leff, 2010: 194), esto nos conduce a resucitar la importancia del concepto de productividad ecotecnológica que articula procesos de productividad ecológica, tecnológica y cultural (Leff, 2010: 194).

Otros aspectos, sin embargo, de dicha estrategia política se pierden cuando se insiste en que se trata de un discurso que deconstruye «el conocimiento disciplinario, simplificador, unitario» (Leff, 2010a: 191), y se olvida señalar aspectos específicos de la manera que debemos organizar las unidades productivas o sus territorios.

En los últimos años, Leff ha reconstruido el discurso ambientalista latinoamericano, producción teórica esencialmente «suya», que se remite a una vuelta a la "pregunta por el ser: el ser del ente, de las cosas, del mundo, del ser bumano» (Leff, 2009: 42), ${ }^{15}$ o sea, a partir de un vocabulario que se dice procede de Heidegger, insistiendo en que el «ambiente» no

[... $]$ es sólo el mundo «de afuera», el entorno del ser y del ente, o lo que queda fuera del sistema de un sistema. El ambiente es un saber sobre la naturaleza externalizada, sobre las identidades desterritorializadas (Leff, 2010a: 190).

${ }^{13}$ «El ambiente no es un objeto perdido en el proceso de diferenciación y especificación de las ciencias, ni un espacio reintegrable por el intercambio disciplinario de los saberes existentes. El ambiente es la falta incolmable del conocimiento; ese vacío en el que anida el deseo de saber, generando una tendencia interminable hacia el desarrollo de las ciencias, el equilibrio ecológico y la justicia social» (Leff, 2007: 90). ${ }^{14}$ «Busca (dice Leff) la recuperación del sentido» (Leff, 2010: 183); «subvierte el logocentrismo y deconstruye el círculo cerrado de las ciencias» (Leff, 2007: 184).

${ }^{15}$ Ver , por ejemplo, Leff (2011: 94). 
Y al mismo tiempo, intenta pensar al «ser» y la gestación de las identidades sin aludir a las asimetrías de poder que señalaban o daban indicios sobre el lugar donde se ejercían los patrones de la organización o del manejo integrado de recursos; señala la importancia del diálogo de saberes (Leff, 2010a: 117), los cuales suponen un mundo entre el ser y el saber, suponiendo la existencia de un ámbito político y territorial por conquistar, precisamente como sus ejemplos demuestran: los zapatistas y los sengueiros. Pero en última instancia las estrategias ecotecnológicas y del desarrollo sustentable suponen diversas formas asimétricas de articular y de poseer en separación ciertas condiciones de existencia de las unidades o espacios territoriales. Leff tiene presente que debemos buscar y concretar un mundo de pluralidades, productivas e identitarias, ya que la racionalidad ambiental se rige por variadas y lógicas contrapuestas.

Es de resaltarse que no obstante haber iniciado con nociones de poder y de distribución y explotación, vocabulario que se hereda del marxismo, habiendo superado dicha perspectiva dadas las inconsistencias de sus formas de plantear el proceso de trabajo, y la posesión en separación de las condiciones de existencia que hacen factible la organización productiva, ${ }^{16} \mathrm{hizo}$ posible al mismo tiempo la crítica a otras nociones generales sobre el valor de los recursos productivos, manteniendo la imposibilidad de generalizar una propuesta, en singular, sobre cómo resolver la manera de organizar, administrar los recursos productivos o la posesión en separación de las condiciones de existencia de los mismos. Incluso niega pertinencia a la analogía que hace Martínez-Alier (2005) sobre los procesos y la distribución de los costos entre las nociones de distribución marxistas o pensamiento neoclásico con su idea de los costos ecológicos:

La categoría de distribución ecológica resulta inespecífica para comprender los conflictos ambientales y ecológicos generados por el impacto de la economía sobre el ambiente y la calidad de vida de la gente [... ] El conflicto ambiental [se] plantea así en un campo estratégico y político heterogéneo donde se mez-

${ }^{16}$ «No se trata de acabar con el comercio, siempre y cuando el intercambio de excedentes esté guiado por valores humanos y políticos» (Leff, 2010a: 85, subrayados míos). 
clan intereses sociales [...] donde lo «ecológico» puede quedar subordinado [... ] a demandas de autonomía cultural (Leff, 2010: 65).

Pero en ocasiones es precisamente este aspecto de la teorización política la que se extravía bajo el velo del «ser ahí» heideggeriano y ya no se diga de la metáforas a partir de la obra de Lacan para pensar la «incompletitud»del ser (Leff, 2011: 130). ${ }^{17}$ Además, debemos subrayar que no hay que olvidar que entre la lógica de la «flecha del tiempo» hacia la entropía, a pesar de existir algunas tendencias «neguentrópicas», ${ }^{18} \mathrm{el}$ mundo como los entendemos va a la muerte: los ritmos y tiempos dispares entre poderes asimétricos, centro y periferia, ciclos bióticos y los financieros no tienen forma de balancearse como a veces cree Leff, a no ser que se repita sin cesar y se intente imponer - a los pueblos y comunidades del sur-, las estrategias de crecimiento del norte a sus ritmos y transformaciones sociales, acorde a las estrategias verdes y la tecnología «limpia» generadas por el coloso del norte.

\section{LOS EXCEDENTES DEL PODER}

Antes de finalizar, y destacar la importancia del surgimiento de varios pueblos y comunidades indígenas y sus respectivas identidades, en su lucha por transformar sus condiciones de existencia, mencionemos algunos conceptos que podrían apoyar los supuestos teóricos sobre una alternativa productiva y ambiental. En ocasiones la lógica del argumento de Leff supone que las comunidades buscan «apropiarse» de la «naturaleza» (Leff, 2010; 64; 2007;

\footnotetext{
${ }^{17}$ Por ejemplo, capítulos 16, 17, 18 y 19, en (Leff, 2010) y capítulo 2 en (Leff, 2013); tercer capítulo en (Leff 2011).

${ }^{18}$ «Ello implica llevar la economía hacia un estado de balance entrópico-neguentrópico. [...] La única posibilidad de detener el colapso ecológico inducido por el proceso económico es la construcción de una nueva racionalidad productiva, fundada en el incremento de la productividad neguentrópica basada en la fotosíntesis - el único proceso neguentrópico del planeta-, que depende de la conservación y restauración de la organización ecosistémica basada en los potenciales de la naturaleza y de la cultura» (Leff, 2010a: 75).
} 
358; 2010a: 85), lo cual podría obstaculizar la idea de que se trata de aspectos sobre la reorganización productiva y regional, o como también dice Leff: «una nueva teoría de la producción y su puesta en práctica en proyectos alternativos de desarrollo» (Leff, 2010: 194), «internalizando» (Leff, 2010: 273) las condiciones «ecológicas del desarrollo sustentable». Proponemos a continuación una serie de indicaciones en torno a los aspectos asimétricos del poder entre distintas unidades o agentes productivos, aspectos que forjan identidades y formas de lucha por la defensa de sus condiciones de existencia que hacen factible la generación de ciertos recursos productivos.

Hay que tomar en serio, como dice Leff, que lo «local es el punto de encuentro de procesos sincrónicos y diacrónicos, donde se precipitan tiempos y momentos diferenciados; es el lugar en donde se articulan culturas y potencialidades ecológicas» (Leff, 2010: 85), lo cual nos obliga a reflexionar sobre las relaciones y circuitos productivos entre diversas unidades productivas o sus agentes.

En referencia a la propuesta de Martínez-Alier sobre los «conflictos de distribución ecológica», Leff dice que se trata de «estrategias de poder — resistencia y negociación- [...] de intereses sociales y políticos que no permiten dirimir los conflictos ambientales en términos estrictamente económicos» (Leff, 2010: 64), y ante la ausencia de un «mecanismo que internaliza los costos ecológicos llevándolos a su justo valor» (Leff, 2010: 64) y ausencia de normas internas de la «economía ni de la ecología que permitan zanjar la cuestión de la justicia ambiental, ya que no son valores estrictamente económicos ni exclusivamente ecológicos los que definen los "costos" y los sentidos movilizadores en defensa de la naturaleza» (idem), estamos obligados a buscar alternativas conceptuales para pensar la manera en que se da articulación entre unidades productivas y sus agentes.

En este ámbito el pensamiento estructuralista latinoamericano ofrece opciones teórico-políticas de avanzada. ${ }^{19}$ Teoriza la conformación de

${ }^{19}$ Véase la más reciente reiteración del estructuralismo latinoamericano en la obra de Armando Di Filippo (2013) y examen en Mallorquin (2013a). 
precios, costos e intercambios a partir de las asimetrías de poder entre los diversos agentes y unidades productivas: niega la pertinencia explicativa de la teoría del valor trabajo (marxista) o subjetiva (neoclásica o marginal), así como la noción del mercado como una entidad unitaria bajo una lógica general; donde de hecho presenciamos la articulación de ciertos efectos de las asimetrías de poder entre los agentes productivos y de diversa índole organizativa.

En dicha concepción, las relaciones sociales y la manera en que organizan los procesos productivos, conjuntos sociales que no están predeterminados tecnológicamente, son consecuencia de las asimetrías y diversos grados de poder entre las unidades de producción y sus correspondientes agentes antagónicos, ya sea debido a sus idearios o vocabularios específicos, o por la defensa de sus condiciones de existencia. Por lo tanto, la economía no es algo independiente de los agentes o algo externo (o fuera) a los mismos. Los aspectos centrales de la teorización estructuralista de la economía son las relaciones sociales. Por eso se impone una perspectiva multidimensional para explicar y plantear alternativas de transformación y superación de las asimetrías de poder. Lo cual implica que en ocasiones la perspectiva latinoamericana propone la «construcción/constitución» de mercados en lugar de formas colectivas o estatales de organización. Ello obedece a que el objetivo es la superación/transformación de las asimetrías de poder entre los agentes en cuestión, es decir, empoderar a ciertos agentes y deconstruir las injusticias y desigualdades:

[...] las reflexiones de Marx sobre la estructura económica son ampliamente aplicables a la noción general de posiciones de poder sustentada aquí. Sin embargo, hemos sustituido la noción marxista de explotación por la noción más amplia de dominación. Ambas suponen estructuras sociales asimétricas, pero no toda forma de asimetría es injusta (Di Filippo, 2013: 99).

El desplazamiento y superación (en términos hegelianos) de la teoría de Marx sobre la explotación y dominio, se hace posible una vez que 
se ha demostrado la inoperancia teórica de la teoría valor-trabajo en Marx. Con la intención de seguir exponiendo la postura de Di Filippo, pero alejándonos un poco de su estrategia crítica, es suficiente decir que el aspecto de explotación en Marx se realiza a través de la acción extractiva de plustrabajo durante el proceso de trabajo (D-M-D). Como toda mercancía, los productos en el mercado ${ }^{20}$ se intercambian por sus equivalentes valores-trabajos («fuerza de trabajo abstracta socialmente necesaria») para su generación. El dinero es solamente un representante de una unidad proporcional de fuerza de trabajo, mediando las ventas y compras en el mercado, ahí no puede surgir una ganancia ( $D-M-D)$.

Por consiguiente, se puede plantear que los precios obedecen a diversas y antagónicas asimetrías de poder, entre las unidades productivas: acceso al crédito, calidad real o virtual de sus bienes, la presencia de cierta concurrencia, y los cálculos a corto o largo plazo de las unidades productivas que a su vez dependen de las anteriores condiciones. El establecimiento de los precios es una forma - entre otras - de ejercer cierto poder por parte de ciertas unidades productivas, de comandar ciertos recursos: cosas y hombres. Pero en

[...] una teoría del poder y de la dominación [...] [e]l titular del poder es el propietario de riqueza. Cuando el propietario de riqueza ejercita su poder, [...], dicho poder recae sobre el proveedor potencial de trabajo [...], y la relación estructurada de poder [...] se verifica a través del mercado, mientras que los fines del dominador [...] pueden ser muy variados; por ejemplo, los que ejemplifica el propio Adam Smith, consistentes en la adquisición de otras formas de poder político, civil o militar. [... L La fuente del poder (la riqueza) posibilita una relación de dominación donde el dominador controla el trabajo del dominado (Di Filippo, 2013: 92-93). [... ] Así, el ejercicio de la capaci-

\footnotetext{
${ }^{20}$ «Si a la demanda agregada se la hace depender de la distribución personal-familiar del ingreso y se la independiza de la ley del valor como fundamento de los precios de mercado en el sentido de Marx, se hace viable considerar los fenómenos de dominación-explotación que se verifican en el mercado [... A Así, el mercado puede ser la fuente efectiva de asimetrías de poder que no responden o no se detectan a través de las categorías marxistas, sobre la base de las cuales se ha edificado la noción de explotación de clases.» (Di Filippo, 2013: 186-187).
} 
dad de elección en el mercado es precedido por la posesión de poder adquisitivo general, requerido para transar todo tipo de mercancías (Di Filippo, 2013: 84) [...] El ejercicio del poder es siempre una categoría relacional, sea que se proyecte sobre las cosas (caso en que hablamos de posesión, producción, consumo, etcétera), sea que se proyecte sobre las personas, en cuyo caso hablamos de dominación (Di Filippo, 2013: 85, subrayados míos).

Subrayo relacional porque se trata de relaciones de poder contingentes, que siempre se encuentran en proceso de transición, de cambio. Por lo tanto, tampoco puede haber una teoría general de la ganancia: las diferencias organizativas y de poder entre las unidades productivas manifiestan sus diversas «condiciones de existencia», es decir, las asimetrías de poder entre sí para poder establecer ciertos precios, generando lo que se ha construido teóricamente como la heterogeneidad (Cfr., Mallorquin, 2011).

Tampoco se trata de negar la coexistencia de una aglomeración de signos monetarios, aludido como «demanda» (pendiente negativa, hacia abajo), ${ }^{21}$ ni el del aglutinado de recursos-bienes, llamado la «oferta» (pendiente positiva, hacia arriba). Se niega la necesaria y univoca intersección entre ellas en un punto que supuestamente refleja un óptimo eficiente, que a su vez se reflexiona independientemente de las relaciones sociales (poder), es más, la heterogeneidad productiva señala varias posibles intersecciones de las curvas, que son finalmente consecuencia de las asimetrías de poder de las unidades productivas entre sí. En otras palabras, se rechaza la existencia de un equilibrio, o en términos de Di Filippo, el mito del capitalismo o mercado autorregulado. ${ }^{22}$ La estabilidad relativa de los precios e ingresos en diversos periodos es consecuencia de la hegemonía de las prácticas contables institucionalizadas, de algunas unidades productivas, entre el universo de ciertos sectores y ramas productivas.

${ }^{21} \mathrm{El}$ consenso y certeza en la academia anglosajona sobre la pendiente en cuestión no estaba dada necesariamente de antemano, la lucha teórica sobre la generación de la hegemonía en cuestión, desde otra narrativa histórica; véanse los trabajos de (Mirowski y Wade, 2006).

${ }^{22}$ La realidad de la economía, el de sus ciclos, su movimiento «ondulatorio» dice Prebisch, se debe a la sistemática «asincronía» (termino de Di Filippo, 1981a: 54) o «disparidad de tiempos» (término de Prebisch, 1949a en 1993: 416) entre el circuito productivo y circulatorio. 
Por lo tanto, ante la ausencia de una teoría general del valor, las remuneraciones entre los agentes de producción se convierten en un ámbito de reflexión sobre el antagonismo y la negociación. Siguiendo a Di Filippo, no todas las asimetrías de poder son «injustas»; las retribuciones y nivel de ingreso estarán limitadas por ciertos patrones y cálculos tradicionales a partir de cierta base mínima de necesidades básicas. Para evaluarlas, ya existe todo un repertorio contable de los sistemas de cuentas nacionales y unidades de cuenta (moneda de cuenta, dice Di Filippo), para hacer comparables o realizar ciertos tipos de equivalencias entre diversos niveles de ingreso y diversas clases y sectores sociales, utilizando a la familia como el centro de atención. Igualmente recordemos que el patrimonio y valor de los recursos productivos renovables y no renovables serán siempre un centro de confrontación y de lucha, como ha insistido Leff.

Se supone un universo social antagónico y desigual, que presenta diversas formas de posesión en separación de las condiciones de existencia de las unidades productivas y las de la mano de obra articulada a las mismas:

Estas formas de explotación cruzan transversalmente todas las clases sociales agrupadas, no por la fuente de donde vienen sus ingresos sino por la magnitud relativa de sus ingresos familiares y personales. Especialmente en las sociedades periféricas contemporáneas (y de América Latina en particular), en los estratos medios y bajos de ingreso encontramos micro y pequeños empresarios o pequeños propietarios rurales, junto con profesionales independientes por cuenta propia, cuyos ingresos respectivos para consumo personal o familiar no difieren sustancialmente entre sí ni respecto de los percibidos por asalariados que venden su fuerza laboral. Todos ellos considerados como personas o como grupos familiares, se ubican en parecidos estratos en materia de distribución personal/familiar del ingreso (Di Filippo, 2013: 87).

Aparentemente, ciertas fuentes principales de explotación radican en los dispares desniveles de ingresos entre personas y grupos familiares, o la injusta distribución funcional del ingreso, estructurado socialmente 
a través de la figura del propietario. Si bien, «todo capitalista es un propietario, no todo propietario es un capitalista» (Di Filippo, 2013:242);23 «[s] olamente los propietarios de alguna mercancía (incluyendo el dinero) pueden participar por derecho propio en los mercados» (Di Filippo, 2013: 74).

Surge entonces la duda sobre las condiciones de existencia del mecanismo de explotación y, por tanto, de la desigualdad e injusticia. Recordemos que ya no podemos apoyarnos en la acepción de extracción de plustrabajo en y durante el proceso de trabajo, como se presentaría bajo la visión marxista. Por lo tanto, la transformación de las relaciones sociales para constituir una sociedad bajo un orden de justicia distributiva, debe retornar a considerar las condiciones de existencia de las asimetrías de poder, las cuales en parte se presentan por los diversos grados de empoderamientos de los agentes y unidades productivas entre sí. Un aspecto es la manera en que se sostiene la defensa de ciertas tasas salariales y el nivel de las canastas básicas mínimas, otro tanto se refiere a un elemento, no contable, que sostiene y da vida a la explotación y desigualdad fundacional originaria: las formas de posesión en separación de las condiciones de existencia de las unidades productivas y de la mano de obra. Es precisamente la posesión en separación de ciertas condiciones de existencia de la producción por parte de cada unidad productiva, el mecanismo que genera las asimetrías de poder: tanto las unidades productivas como la fuerza laboral, poseen en separación únicamente algunas de las condiciones de su reproducción: esa carencia se resuelve por medio del intercambio, del proceso de circulación, en otras palabras por medio del mercado. Las relaciones mercantiles obedecen

\footnotetext{
${ }^{23}$ «Los valores básicos de la democracia se ven de manera diferente, según se miren con los ojos de propietarios o con los de ciudadanos. Los primeros se pueden definir como titulares de derechos y obligaciones de naturaleza patrimonial; los segundos, como titulares de derechos y obligaciones de naturaleza civil (política o cultural)» [...] «En la práctica, el tema de la condición de propietario se impone y desplaza la condición de ciudadano. Ese desplazamiento implica un desplazamiento de los derechos humanos y ciudadanos de los carentes de propiedad. La relación entre libertad y propiedad es por lo tanto también una relación entre el concepto político de ciudadano y el concepto económico de propietario» (Di Filippo, 2013: 71 y 72).
} 
su existencia a dicho resquicio, a los elementos que no pueden ser cubiertos por una misma unidad productiva, requiere de su articulación a otras unidades productivas por medio de un sistema de equivalencias negociadas, establecimiento de precios, políticas de inversión y de endeudamiento, tasas salariales: las diversas condiciones de existencia para cubrir íntegramente los aspectos de la producción, aspectos que generan la intrínseca heterogeneidad e inestabilidad del capitalismo (tanto en el centro como en la periferia).

A su vez, esto implica que las relaciones de poder, las formas de posesión en separación entre las unidades productivas y la fuerza laboral sean de hecho el mercado. Utilizando una expresión de Di Filippo quiero resaltar una importante diferencia para pensar el sustrato principal de la generación de las asimetrías de poder. Di Filippo menciona que «[...] el tema de la justicia distributiva está detrás del mercado y tiene relación con la naturaleza de las instituciones que determinan esa distribución y con el contenido moral de las decisiones de cada demandante» (Di Filippo, 2013: 234, subrayados míos):

Sugiero mejor que el mercado no está fuera ni detrás, ${ }^{24}$ de los agentes productivos como podría ser el caso de aquella muchedumbre o localidad geográfica que mencionamos coloquialmente como mercado a unas cuadras de nuestros hogares: las asimetrías de poder y por tanto la explotación, se debe a esa desarticulación de las unidades productivas entre sí y la fuerza laboral respectiva, es decir, el mercado, implica esa desarticulación potencial, que se resuelve con la compra y venta de mano de obra y productos. En otras palabras, a través de la circulación mercantil. La estrategia política de transformación de las asimetrías de poder recae, en un primer momento, en otorgar y crear cierta institucionalización del uso de la unidad de cuenta, o moneda de cuenta como

\footnotetext{
${ }^{24}$ «Para mí el mercado no es bueno ni es malo, ni es justo o injusto, son las fuerzas que están detrás del mercado las que le imprimen su carácter. Si hay una distribución desigual de la que sale una demanda desigual que favorece a ciertos grupos sociales en desmedro de otros, naturalmente que el mercado funciona mal; pero no es el mercado en sí, son las fuerzas que están detrás de él (Prebisch, 1982: 105-106, subrayados míos).
} 
dice Di Filippo, pero reduciendo o limitando su radio de acción y usos alternativos fuera del ámbito productivo propiamente dicho ${ }^{25}$ Además de constituir las canastas básicas, se requiere generar otros mecanismos de articulación entre las unidades productivas entre sí y la fuerza laboral, además de la moneda de cuenta. Incluso en las más modernas economías, los niveles salariales y el valor de la moneda de cuenta forma parte de un proceso negociado vis-à-vis otros Estados o economías.

Sin embargo, incluso en el caso más utópico y extremo de socialización o articulación de las condiciones de existencia de las unidades productivas entre sí a través del plan (Bettelheim, 1975), se requiere el uso de una especie análoga de relación salarial y el uso de una moneda de cuenta, es decir, será necesario el curso de cierto instrumento para realizar o construir equivalencias (Di Filippo, 1981a), suponiendo la presencia de cierta mercantilización del imaginario social. La superación de la posesión en separación, a través del plan respecto la totalidad de las condiciones de existencia en la Unión Soviética, fue quizá uno de los sueños de la razón más atolondrados de reciente data. El o los mercados somos nosotros, los agentes productivos, y llegaron para quedarse. No existe nada detrás del mercado de donde cabe deducir el poder y sus asimetrías, es la separación en posesión de ciertas condiciones de existencia por parte de ciertas unidades productivas respecto de las necesidades de otras unidades y la mano de obra lo que constituye la fuente de la explotación y por tanto la fuente de la asimetría de poder. Igualmente, ello supone que las clases como categorías económicas no desaparecerán y tendremos que definirlas en tanto su específica posesión en separación de algunas de las condiciones de existencia para la reproducción de las mismas y de la totalidad social. Algunas unidades monopolizarían

${ }^{25}$ Actualmente de difícil materialización en el ámbito internacional dado la financiarización tan en boga y que supera cualquier tipo de «regulación» en proceso; en pocos años pasamos de Basilea I a Basilea III, en parte, dado al dominio de una desregulación internacional que nadie sabe cómo frenar, que se denota, por ejemplo, en la utilización de derivados para «fortalecer» las finanzas y «portafolios» de ciertas unidades productivas y otras no tanto, como las propias ¡«financieras»! (Soto, 2010). 
ciertas condiciones, inmersas en un mar de pequeñas unidades (Pymes) productivas - a veces no tan pequeñas-, las cuales jurídicamente podrían constituirse en cooperativas o «empresas pequeño burguesas», como solía decirse, dependiendo de la amplitud de las condiciones de posesión en separación (la heterogeneidad de la cual habla el estructuralismo latinoamericano). Por lo que hemos estado argumentando, la problemática de la heterogeneidad no es meramente un aspecto tecnológico, o de productividad, sino de poder, debido a que la medición de la productividad depende a su vez de la manera en que podemos establecer cálculos y equivalencias a corto y largo plazos. En este sentido, siempre existirán formas de separación en posesión entre diversas unidades de producción, generando asimetrías de poder tanto por la forma de articularse entre sí - definidas por ciertas reglas-convenio o simplemente desreguladas - o debido al tipo de acceso a la generación de dinero-crédito, con sus respectivas capacidades de negociar salarios-precios. El establecimiento de equivalencias, de precios, se convierte en un proceso de reflexión y cálculo político, aspecto teórico que antes se suponía resuelto citando a la teoría general de valor-trabajo o utilidades.

La problemática regional y transectorial (Leff, 2007:301; 2010a: 131) que menciona Leff, también será un ámbito de antagonismos $y$ confrontación entre los diversos agentes y unidades de producción y regiones. No será un espacio libre de la lucha política a no ser que otorguemos, sin discusión, algún tipo de privilegio o principio de intervención extraterritorial a ciertas comunidades vis-à-vis otros pueblos o al Estado: una especie de apartheid productiva auto-organizada que tampoco es la solución para algunos pueblos que han generado tecnologías de comercio ${ }^{26}$ o dispositivos de intercambio, formas híbridas para establecer ciertas equivalencias, entre reciprocidades y cálculos monetarios como defensa de sus recursos productivos de ciertos circuitos mercantiles o del mercado. Asimismo, se ha puesto mucho énfasis teórico en los aspectos asimétricos de poder entre los agentes y las unidades pro-

${ }^{26}$ La noción de technological trade la desarrolla De Vroey (2004). 
ductivas para calcular aspectos micro y macroeconómicos, porque serán esas lógicas las que sobre determinarán al proyecto ecotecnológico a pesar de la entropía consustancial del mismo. Únicamente los países del Norte pueden darse el lujo de algo llamado «crecimiento cero», no los de la periferia. ${ }^{27}$

No podemos negarle a Leff el reconocimiento del mestizaje teórico excepcional que reflejan sus reflexiones, subrayando los trópicos y su biodiversidad: el haber construido una perspectiva latinoamericana para la praxis medioambiental. Los conceptos híbridos y el mestizaje teórico reflejan la manera en que transformó el discurso del Norte demarcando el pensamiento ambientalista latinoamericano y la ecología; no obstante, nos recuerda los senderos y vocabularios que tuvo que desenmarañar (Leff, 2010a: 244; 2006), que inicialmente proceden de varios teóricos y profesores de su época parisina, pero también subraya y destaca su distanciamiento «epistemofílico» (Leff, 2010a: 242) que obedece al proceso que generó una perspectiva ambiental latinoamericana, con identidad propia ${ }^{28}$ Leff nos ofrece varios itinerarios de salida para librarnos del laberinto del desarrollo sustentable y la complejidad ambiental, pero en algunos casos no será debido al vocabulario sobre el «ser en el tiempo» sino a la generación de proyectos antagónicos del ser y sus estrategias, y saberes de poder específicos. En el transcurso de la lucha y la organización, por formas de reconstrucción productiva surgirán periodos de decisiones difíciles, producto de discusiones democráticas, que no podrán defenderse en equilibrios ecológicos reales o imaginarios sobre la naturaleza. ${ }^{29}$ Por lo mismo creo que el invocar un vocabulario

\footnotetext{
${ }^{27}$ En Mallorquin (2013b), se intenta desarrollar esta problemática.

${ }^{28}$ «Estas nuevas perspectivas epistemológicas surgieron en América Latina; no se produjeron en Europa como un desarrollo del racionalismo crítico francés [... A A pesar de su indudables sintonías con el pensamiento complejo que surgía en esos tiempos en la obra de Edgar Morin, la producción del concepto de ambiente fue adquiriendo una identidad propia» (Leff, 2010a: 242).

${ }^{29}$ Desafortunadamente los efectos del saber/poder tanto hoy, como en el pasado, supone una especie de electiva «autodestrucción» creativa, lo cual supone institucionalizar mecanismos para que surja a partir de nuestras propias elecciones: uno entre tantos ejemplos, puede señalarse el surgimiento del «evolucionismo darwiniano»; como lo señala A. Ashwell: «Pero el planeta y los animales,
} 
heideggeriano para explicar y plantear las políticas de identidad de los agentes económicos o las comunidades es relativamente irrelevante: porque en última instancia no se puede eludir la decisión y las consecuencias de que una política de desarrollo, desde los pueblos y las naciones del Sur supone dispositivos que generen crecimiento y destrucción de los recursos renovables y no renovables, anhelando que la evolución se dé bajo la hegemonía de sus autóctonas formas de vida. No es por tanto una cuestión que pueda sustentarse o resolverse con «epistemologías» de ninguna índole, como si los discursos substantivos y sus vocabularios fueran productos de alguna en particular (Rorty, 1979; Davidson, 2001; Lander, 2000).

Tampoco se trata del retorno de la clásica oposición entre mercado o Estado, sino la de reconstruir, transformar, las asimetrías de poder entre las comunidades y agentes productivos, partiendo de las diversidad simbólica y heterogeneidad productiva; de hecho en algunas comunidades de la región comercian «hacia afuera», ya que «hacia adentro» las equivalencias y los intercambios proceden de una serie de prácticas y costumbrescriterios de otra índole, subordinando a las categorías monetarias.

En ocasiones se trata de las atribuciones que posee un Estado para articular entre sí diversas unidades, comunidades y municipios en la redistribución de ciertos recursos, en otras, por la propia asociación electiva entre las comunidades entre sí, respecto de las acciones del Estado si fuera necesario. En otras palabras, se trata de la reconstitución de una serie de lazos y tecnologías de intercambio. Recordemos que la hegemonía del neoliberalismo se instauró llamando por la desregulari-

atormentados y perseguidos por la arrogancia interrogativa de los evolucionistas, también dieron cuenta de nuestra humanidad perdida: el hombre, cuando más conocimiento adquirió sobre sus orígenes biológicos ligados al origen de todos los demás seres vivos en el planeta, más se apartó de una habitación ética con los otros animales y la naturaleza. Con la explicación científica de la presencia humana en una larga historia evolutiva se había - como explican algunos filósofosdistorsionado esencialmente no sólo el lugar del hombre en la naturaleza sino derechos que éste se adjudicó sobre el otro mundo animal. La pregunta por los pájaros — persiste y se acrecienta el exterminio de la biodiversidad y aumenta la tortura y el mal trato de los animales, también en nombre de la ciencia - apunta a una evolución en el cual la humanidad del hombre está oculta»» (Ashwell, 2009: 12). 
zación del ámbito económico y social. Antes de terminar, leo sobre el derrame de 40,000 metros cúbicos de sulfato de cobre al río Sonora, procedente de una de las mineras más grandes del mundo y la de mayor tamaño en México, y se escucha decir que la Secretaría de Economía tiene atribuciones para la cancelación del permiso de explotar los recursos de la zona... ¿será?

En el presente texto se ofreció una síntesis de la evolución del vocabulario de Enrique Leff en torno a la cuestión ambiental, subrayando el tránsito y recorrido de la evolución discursiva que culminó en un mestizaje teórico regional, latinoamericano. No obstante, a medida que se desplaza la noción marxista de la economía, se va generando una importante ausencia o falta: planteamientos en torno a la reorganización de los recursos productivos y las asimetrías de poder de los agentes y comunidades en cuestión, lo cual se intenta subsanar con la subsunción de su perspectiva bajo otra perspectiva de procedencia regional sobre la economía: el estructuralismo latinoamericano, teorización de la organización productiva que toma como punto de partida las asimetrías de poder para pensar la producción y la distribución de los recursos, perspectiva que va más allá de la común acepción de que se trata de una teoría de la industrialización de la región. Muy al contrario, la concepción del desarrollo en cuestión implica pensar las particularidades sociales que sustentan las asimetrías de poder de los agentes, regiones y naciones entre sí. Por lo mismo, siempre rehusó la distinción u oposición naturaleza/cultura para pensar alternativas de producción y así distinguir el proceso históricamente. Sin embargo, hay que subrayar que los imperativos teóricos que proceden de la perspectiva para reflexionar la heterogeneidad no supone, necesariamente, el imperativo ético o político de buscar formas comunitarias o socializadas de producción, ello será resultado de una decisión personal, lo cual explica a su vez la diversidad política que presentan los autores que se dicen estructuralistas a partir de la obra de Raúl Prebisch. ${ }^{30}$

\footnotetext{
${ }^{30} \mathrm{El}$ vocablo «estructuralismo latinoamericano», su aparición y significado en la región no tiene nada que ver con el vocablo «estructuralismo» de procedencia francesa o europea de la década de los años sesenta; véase Mallorquin (1998).
} 


\section{BIBLIOGRAFÍA}

Althusser, Louis, Balibar Etienne (2004 [1967]), Para leer El Capital, México, Siglo XXI.

Ashwell, Anamaría (2009), «En el bicentenario de Darwin, ¿y los pájaros?», Elementos, vol.16, núm., 74, pp. 3-13.

Bettelheim, Charles (1975), Economic Calculation and Forms of Property, New York, Monthly Review Press.

Davidson, Donald (2001), Inquiries into Truth and Interpretation, U.K, Oxford University Press.

De Vroey, Michel (2004), «The History of Macroeconomics Viewed against the Background of the Marshall-Walras Divide», en De Vroey, Hoover Kevin D. (eds.), The IS-LM Model: Its Rise, Fall, and Strange Persistence, Durham \& London, Duke University.

Di Filippo, Armando (2013), Poder, capitalismo y democracia. Una visión sistémica desde América Latina, Santiago de Chile, RIL Editores.

(1981a), Desarrollo y desigualdad social en la América Latina, Lecturas, núm. 44, México, Fondo de Cultura Económica.

Foladori, Guillermo (1996), «La cuestión ambiental en Marx», Revista de Ciencias Sociales, núm. 12.

Gallopin, G., (2000), «Ecología y ambiente», en Leff, Enrique (2000), op. cit.

Herrera, A. O, et al. (1976), ¿Catástrofe o nueva sociedad. El modelo mundial? (primero en inglés en 1976), editor: Centro Internacional de Investigaciones para el Desarrollo (en español, 1977).

LAnder, Edgardo (coord.) (2000), La colonialidad del saber, Buenos Aires, Clacso/ Unesco.

LefF, Enrique (1980), «La teoría del valor en Marx frente a la revolución- científico-tecnológica», en Enrique Leff, (ed.), Teoría del valor, México, unam.

(2013 [2004, $\left.\left.1^{\mathrm{a}} \mathrm{ed}.\right]\right)$, Racionalidad ambiental. La reapropiación social de la naturaleza, México, Siglo XXI.

(2007 [1986, $1^{\text {a }}$ ed.]), Ecología y capital. Racionalidad ambiental, democracia participativa y desarrollo sustentable, México, Siglo XXI.

(coord.) (2000 [1986, $1^{\mathrm{a}} \mathrm{ed}$.]), Los problemas del conocimiento y la perspectiva ambiental del desarrollo, México, Siglo XXI.

(2009), «Pensar la complejidad ambiental», en Enrique Leff, (coord.) (2009 [2000]), La complejidad ambiental, México, Siglo XXI.

(2010 [1998, $1^{\text {a }}$ ed.]), Saber ambiental. Sustentabilidad, racionalidad, complejidad, poder, México, Siglo XXI. 
(2010a), Discursos sustentables, México, Siglo XXI.

(2011 [2006, $1^{\mathrm{a}}$ ed.]), Aventuras de la epistemología ambiental, México, Siglo XXI.

Mallorquin, Carlos (1998), Ideas e historia en torno al pensamiento económico latinoamericano, México, Plaza y Valdés.

(2013), Relatos contados desde la periferia: el pensamiento económico latinoamericano, México, Plaza y Valdés.

(2013a), «América Latina y su teoría», Estudios Críticos del Desarrollo, vol. III, núm. 5 .

(2013b), «Las antinomias del desarrollo social sustentable en la periferia», en Margarita González, Darcy Tetreault y Humberto Márquez, (coords.) (2013), Senderos de la insustentabilidad. Degradación bumana y ambiental en el capitalismo neoliberal, México, Miguel Ángel Porrúa.

Martinez-Alier, Joan (2005), El ecologismo de los pobres. Conflictos ambientales $y$ lenguajes de valoración, Barcelona, Icaria.

Meadows, Donella et al. (1972), Limits to Growth: A Report for the Club of Rome's, Project on the Predicament of Mankind, New York, Universe Books.

Mir Cuerdo, M., J. L. Gorostiza Ramos, (2000), Economía y naturaleza, Madrid, Síntesis.

Mirowski, P. y D. Wade Hands (2006) (eds.), Agreement on Demand: Consumer Theory in the Twentieth Century, (History of Political Economy Annual Supplement), Duke University Press, Supplement edition.

Prebisch, Raúl (1981), Capitalismo periférico. Crisis y transformación, México, Fondo de Cultura Económica.

(1982), «Crisis del capitalismo y la crisis de las teorías económicas», en Fernando Henrique Cardoso, Raúl Prebisch y Rosario Green (coords.), En torno al Estado y al desarrollo, México, Nueva Imagen y Centro de Estudios del Tercer Mundo.

(1993), Raúl Prebisch. Obras 1919-1948, vol. IV, Buenos Aires, Fundación Raúl Prebisch.

([1949a] en 1993), Teoría dinámica de la economía. Conferencias sustentadas por el Sr. Raúl Prebisch en la Escuela Nacional de Economía, (febrero-marzo de 1949), México, Banco de México.

Rorty, Richard (1979), Philosophy and the Mirror of Nature, Princeton, New Jersey, Princeton University Press.

Sото, Roberto (2010), Especulación e innovación financiera, México, Miguel Ángel Porrúa. 
